Reprod. Nutr. Dévelop., 1985, 25 (6), 1029-1035.

\title{
Induction d'une ovulation post-partum chez la vache créole à l'aide d'une séparation temporaire du veau et de I'utilisation préalable d'un implant de Norgestomet
}

\author{
D. GAUTHIER (1), G. COULAUD (1), F. VALLEE
}

Station de Recherches Zootechniques, INRA-CRAAG Domaine Duclos,

97170 Petit-Bourg, Guadeloupe, France.

Summary. Induction of post-partum ovulation in creole cows by using a Norgestomet implant and temporary removal of the calf.

Two treatments were used to attempt to induce ovulation in anoestrous post-partum creole cows.

The use of a Norgestomet implant for 7 days followed by removal of the calf for $48 \mathrm{~h}$ induced ovulation in 91,83 and $71 \%$ of anoestrous creole cows treated respectively at 37 , 48 and 51 days post partum (implant insertion). In the same time, the cycling rates of the control groups were 30,35 and $35 \%$, respectively.

But calf removal for $48 \mathrm{~h}$ at 60 and 80 days without progestagen priming failed to increase the number of cycling cows compared to the control group.

Whatever the treatment and the post-partum day, calf growth was not different between the treated and the control groups at 30,60 and 90 days post-partum, and there was no mastitis.

\section{Introduction.}

De nombreuses études ont montré que la reprise de l'activité ovarienne postpartum était plus précoce chez les vaches traites ou taries que chez celles allaitant leur veau (revue de Peters, 1984). Chez ces dernières, la diminution de la fréquence journalière des tétées entraîne une réduction de l'intervalle vélage1ère ovulation (Randel, 1981) et la séparation des deux partenaires du couple mère veau pendant $48 \mathrm{~h}$ permet $\mathrm{d}$ 'augmenter le nombre de vaches cycliques à un stade post-partum donné (Tervit et al., 1982).

De même, si dans un nombre limité de cas l'utilisation de progestagène chez des vaches en anoestrus semble induire une cyclicité ovarienne (Miksch et al., 1978), bien souvent ce traitement reste sans effet (Smith et Vincent, 1972 ; Smith et al., 1979).

(') Laboratoire de I'Elevage bovin, I.N.R.A., Theix, 63122 Ceyrat, France. 
Chez la vache Créole, la fertilité au cours d'une saison de monte d'une durée limitée est corrélée au pourcentage de femelles ayant une activité ovarienne cyclique au début de cette période (Gauthier et al., 1984a). Ce pourcentage étant très dépendant de la durée de l'anoestrus post-partum, toute technique permettant d'induire une cyclicité précocément après le vêlage devrait entraîner une augmentation de la fertilité des troupeaux. C'est pourquoi l'objectif de cette étude est de diminuer la durée de l'anoestrus post-partum de la vache Créole en la séparant temporairement de son veau avec ou sans traitement préalable par un progestagène.

\section{Matériel et méthodes.}

\section{Environnement et animaux.}

Ces expériences sont réalisées au Domaine de la Station de Recherches zootechniques de I'INRA en Guadeloupe (Gardel). La pluviométrie annuelle est de $1280 \mathrm{~mm}$. Une saison des pluies (août à novembre : $830 \mathrm{~mm}$ ) et une période sèche (décembre à juillet : $450 \mathrm{~mm}$ ) peuvent être définies. La température maximale moyenne varie entre $27^{\circ} \mathrm{C}$ (janvier) et $32{ }^{\circ} \mathrm{C}$ (août), la température minimale entre $21^{\circ} \mathrm{C}$ et $25^{\circ} \mathrm{C}$. L'hygrométrie moyenne est toujours supérieure à $70 \%$.

Les animaux expérimentaux sont des vaches Créoles allaitantes dont les principaux caractères zootechniques ont été décrits précédemment (Gauthier et al., 1984b). Elles sont maintenues toute l'année en savane naturelle ou plantée de Pangola (Digitaria decumbens) et ne reçoivent aucune complémentation minérale. Un mélange mélasse-urée (3\%) est toutefois proposé aux animaux pendant la saison sèche à raison de $2 \mathrm{~kg} /$ jour/tête, lorsque ceux-ci ont perdu en moyenne plus de $15 \%$ de leur poids vif. Les vaches allaitent un veau et un seul durant 7 mois.

\section{Protocoles expérimentaux.}

Expérience 1. - Quarante vaches Créoles vêlant entre le 6-6 et le 29-7 (fin saison sèche) sont divisées en 3 groupes selon leur poids vif et date de vêlage. A $51 \pm 12$ jours post-partum $(m \pm \sigma)$ toutes les vaches du groupe 1 (14) reçoivent un implant silastic de Norgestomet délivrant $200 \mu \mathrm{g}$ de produit par jour (Intervet, Angers). Sept jours plus tard, au retrait de l'implant, les veaux sont séparés des mères pendant $48 \mathrm{~h}$ et maintenus dans un corral où ils disposent d'eau et de fourrage. Pour le groupe 2 (13 vaches), les veaux sont séparés des mères pendant $48 \mathrm{~h}$ à $60 \pm 11$ jours post-partum, comme dans le groupe 1 , mais les vaches ne reçoivent pas d'implant. Le même protocole est appliqué aux 13 vaches du groupe 3 à $80 \pm 13$ jours post-partum, ce lot étant considéré comme témoin des deux autres jusqu'à cette date.

Expérience 2. - Le traitement du lot 1 de l'expérience précédente a été appliqué à $48 \pm 12$ jours post-partum (pose de l'implant) à 17 vaches Créoles vêlant entre le 27-11 et le 31-1 (fin de saison des pluies). Dix-sept autres femelles, vêlant à la même époque constituent le lot témoin. 
Expérience 3. - Quarante-quatre vaches Créoles vêlant entre le 18 mai et le 10 juin (fin de la saison sèche) sont réparties en deux groupes, de 21 et 23 femelles, selon leur poids vif et leur date de vêlage. A $37 \pm 5$ jours postpartum toutes les femelles de l'un des lots $(n=23)$ reçoivent le traitement de l'expérience 2. L'autre lot constitue le groupe témoin ( $n=21$ ).

Pour l'ensemble des 3 expériences les vaches et les veaux sont pesés tous les 28 jours. L'activité ovarienne des femelles est suivie par l'étude de la progestéronémie. Des prélèvements sanguins sont réalisés 2 fois à 10 jours d'intervalle avant la pose de l'implant (Thimonier, 1978), puis une fois par semaine pendant le mois suivant la séparation du veau. Pour une vache donnée, la cyclicité est considérée comme induite par le traitement lorsque celle-ci est en anoestrus avant le retrait du veau et qu'un niveau élevé de progestérone apparaît moins de 4 semaines après. La date d'apparition de ce niveau élevé est définie comme date de mise en place de la cyclicité. Le taux d'induction de l'activité ovarienne est donc pour un lot : le nombre de femelles en anoestrus avant le traitement et cycliques moins de 4 semaines après le retrait du veau/le nombre total de femelles en anoestrus avant le traitement.

De plus l'apparition de mammites est surveillée sur l'ensemble des animaux pendant les 4 semaines suivant le traitement.

\section{Résultats.}

Expérience 1 (fig. 1). - Le pourcentage de femelles cycliques dans le groupe 1 passe de 0 avant la pose de l'implant à $64 \%$, huit jours après le retrait des veaux et à $71 \%$ une semaine plus tard ; cette augmentation est significativement différente $(P<0,01)$ de celle enregistrée pour le lot témoin (groupe 3 ) pendant la même période (de 15 à $38 \%$ ).

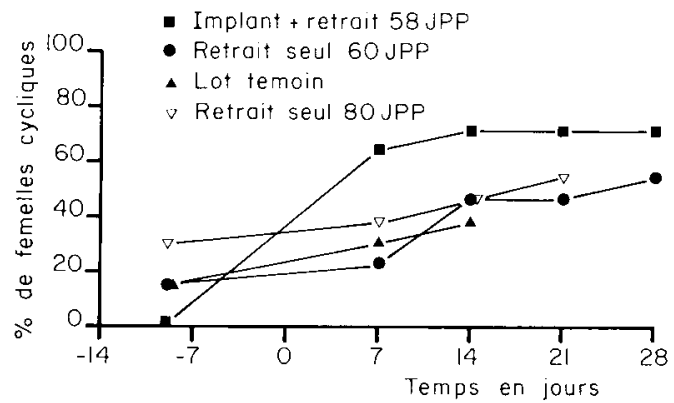

FIG. 1. - Influences respectives d'un implant et/ou du retrait du veau à divers moments post-partum sur la cyclicité des vaches Créoles.

En revanche, dans le groupe 2, I'augmentation du taux de cyclicité (de 15 à 23 puis $46 \%$ ) n'est pas significativement différente de celle du lot témoin.

Pour le groupe 3 , le retrait des veaux à 80 jours post-partum n'entraîne pas d'augmentation significative du pourcentage de femelles cycliques (de 38 à $46 \%)$. 
Le poids moyen des femelles à 90 jours post-partum (non différent entre les lots : $298 \pm 25 \mathrm{~kg}$ ) est bien inférieur à celui obtenu habituellement à cette saison $(330 \mathrm{~kg})$ et est inférieur à celui des femelles vêlant en saison sèche $(312 \mathrm{~kg})$.

Expérience 2. - De 29 et $18 \%$ avant le retrait des veaux, les pourcentages de femelles cycliques passent à 72 et $35 \%$ huit jours après $(P<0,05)$ respectivement pour les lots traité et témoin (fig. 2), par la suite les différences ne sont plus significatives. Le taux d'induction de la cyclicité ovarienne est donc de $83 \%$.

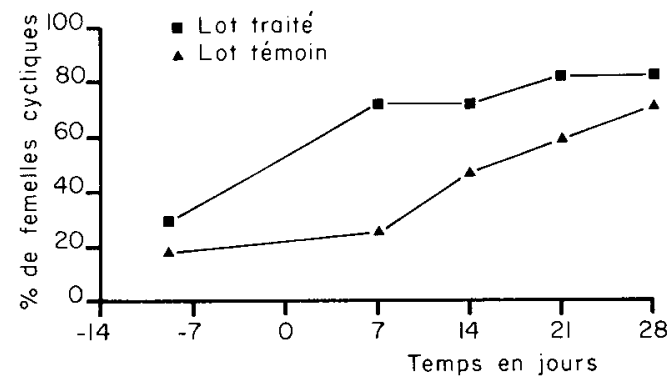

FIG. 2. - Influence d'un traitement progestagène suivi d'un retrait temporaire du veau pratiqué à 55 jours post-partum sur la cyclicité des vaches Créoles.

Les poids des vaches et des veaux ne diffèrent pas entre les lots à 30,60 et 90 jours post-partum (tabl. 1). Le traitement n'a entraîné aucune mammite.

\section{TABLEAU 1}

Poids des vaches et des veaux Créoles à 30,60 et 90 jours post-partum, avec ou sans séparation de $48 \mathrm{~h}$ du couple mère-veau au cours de la lactation $(\mathrm{kg} ; \mathrm{m} \pm \sigma)$.

\begin{tabular}{|c|c|c|c|c|c|c|c|}
\hline \multirow{2}{*}{ Expérience } & \multirow{2}{*}{$\begin{array}{c}\text { Jours post-partum } \\
\text { Lot }\end{array}$} & \multicolumn{2}{|c|}{30} & \multicolumn{2}{|c|}{60} & \multicolumn{2}{|c|}{90} \\
\hline & & Traité & Témoin & Traité & Témoin & Traité & Témoin \\
\hline $\begin{array}{c}3 \\
\text { séparation } \\
\text { à } 44 \text { jours }\end{array}$ & Mères & $325 \pm 32$ & $328 \pm 35$ & $326 \pm 30$ & $328 \pm 34$ & $320 \pm 33$ & $321 \pm 40$ \\
\hline post-partum & Veaux & $48 \pm$ & $48 \pm 10$ & $64 \pm$ & $66 \pm 11$ & $79 \pm 9$ & $80 \pm 15$ \\
\hline $\begin{array}{c}2 \\
\text { séparation } \\
\text { à } 55 \text { jours }\end{array}$ & Mères & $332 \pm 35$ & $325 \pm 41$ & $330 \pm 31$ & $322 \pm 35$ & $316 \pm 38$ & $310 \pm 40$ \\
\hline post-partum & Veaux & $43 \pm$ & $37 \pm 7$ & $58 \pm$ & $54 \pm 8$ & $76 \pm 11$ & $72 \pm 13$ \\
\hline
\end{tabular}

Expérience 3. - Avant le traitement, les taux de femelles cycliques sont de 0 et $5 \%$ respectivement pour les lots traité et témoin. Huit jours après le retrait des veaux ces taux sont de $78 \%$ pour le lot traité et de $10 \%$ pour le lot témoin $(P<0,001)$; la semaine suivante ces chiffres sont respectivement 91 et $33 \%$ pour les 2 lots ( $P<0,01$; fig. 3 ). Le taux d'induction de la cyclicité est donc de $91 \%$ pour le lot traité. 
Les poids des vaches et des veaux ne sont pas différents entre les deux iots à 30,60 et 90 jours post-partum (tabl. 1). Aucune mammite n'est apparue sur les mères à la suite de ce traitement.

Le taux d'induction de la cyclicité suite au traitement complet (progestagène plus retrait du veau) ne diffère pas significativement d'une expérience à l'autre.

Pour l'ensemble de ces expériences le poids vif moyen des femelles dont la cyclicité ovarienne est stimulée par le traitement $(325 \mathrm{~kg})$ est significativement plus élevé que celui des autres femelles $(294 \mathrm{~kg} ; \mathrm{P}<0,05)$.

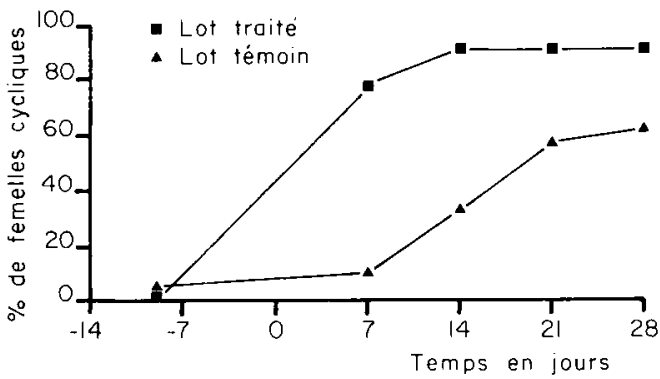

FIG. 3. - Influence d'un traitement progestagène suivi d'un retrait temporaire du veau pratiqué à 44 jours post-parturn sur la cyclicité des vaches Créoles.

\section{Discussion.}

Dans nos conditions expérimentales, la séparation temporaire du veau de sa mère pendant $48 \mathrm{~h}$, même appliquée à un stade post-partum tardif ( 80 jours), est peu efficace lorsqu'elle n'est pas précédée par une application de progestagène. En revanche, l'application d'un implant de progestagène à 50 jours post-partum et pendant 7 jours, suivi d'un retrait du veau pendant $48 \mathrm{~h}$ permet d'induire l'ovulation chez $77 \%$ des vaches traitées quelle que soit la saison. Un traitement plus précoce (37 jours post-partum) est également efficace (91\% d'induction).

Bien que Tervit et al. (1982) diminuent très significativement la durée de l'anoestrus post-partum des vaches en effectuant uniquement une séparation du veau de sa mère, de nombreux auteurs montrent, comme nous, l'absence de véritable efficacité de cette technique (Kiser et al., 1980 ; Smith et al., 1979 ; Walters et al., 1984) et l'attribuent, la plupart du temps, au mauvais état nutritionnel des animaux. Cependant, comme nous le montrons, un traitement complet (progestagène + retrait du veau) même effectué dans de mauvaises conditions alimentaires entraîne une bonne induction de l'ovulation (expérience $1 ; 71 \%$ ). II faut donc penser que la séparation temporaire du veau ne constitue pas une stimulation assez efficace pour obtenir chez la mère un anoestrus post-partum de courte durée. De plus, il a été démontré que l'application du traitement progestagène seul ne constituait pas non plus une stimulation suffisante pour induire l'ovulation (Smith et al., 1979 ; Peterson et al., 1979 ; Pace et Sullivan, 1980). Ainsi la synergie des deux techniques s'avère beaucoup plus intéressante que leur utilisation séparée. Le progestagène agirait en sensibilisant l'ovaire à l'action des gonadotro- 
pines (Smith et al., 1979) sécrétées par la femelle lors de la séparation du veau (Amoss et al., 1981), comme cela semble d'ailleurs être le cas lors de l'induction d'ovulation par « effet mâle » chez les caprins (Chemineau, 1985).

\section{Conclusion.}

L'utilisation d'un implant de progestagène, laissé pendant 7 jours, suivie $\mathrm{d}^{\prime}$ une séparation temporaire du couple mère-veau pendant $48 \mathrm{~h}$ permet d'obtenir une bonne induction de l'ovulation entre 30 et 70 jours post-partum chez la vache Créole. Cette technique peut donc être utilisée dans la pratique courante de l'élevage et cela d'autant plus qu'aucun effet sur la croissance des veaux ou le nombre de mammites chez les mères n'a été mis en évidence.

Reçu en avril 1985

Accepté en juillet 1985.

Remerciements. - Les auteurs remercient D. Aguer (Intervet, Angers) pour le don des produits de traitement et les Drs Thimonier et Matheron pour leurs conseils dans la réalisation du protocole et la rédaction du manuscrit.

\section{Références}

AMOSS M. S., NIX K. J., HARMS P. G., WILTBANK J. M., 1981. Endogenous luteinizing hormone (LH) and prolactin (PRL) release after calf removal in the post-partum cow. Beef cattle research in Texas, 66-69.

CHEMINEAU P., 1985. Effects of progestagen on buck-induced short ovarian cycles in the Creole meat goat. Anim. Reprod. Sci., 8 (sous presse).

GAUTHIER D., COULAUD G., VARO H., THIMONIER J., 1984a. Durée de l'anoestrus postpartum et fertilité de la vache Créole en climat tropical : influence de la saison de mise bas et de la variation du poids vif. Ann. Zootech., 33, 235-244.

GAUTHIER D., AUMONT G., BARRE N., BERBIGIER P., CAMUS E., LAFORTUNE E., POPESCU P., RULQUIN H., XANDE A., THIMONIER J., 1984b. Le bovin Créole en Guadeloupe: caractéristiques et performances zootechniques. Rev. Elev. Méd. vét. Pays Trop., 37، 212-224.

KISER T. E., DUNLAP S. E., BENYSHEK L. L., MARES S. E., 1980. The effect of calf removal on estrous response and pregnancy rate of beef cows after synchromate $B$ treatment. Theriogenology, 13, 381-389.

MIKSCH E. D., MUKEMBO G., LEFEVER D. G., WILTBANK J. N., 1978. Synchronization of estrus in beef cattle. II. Effect of injection of norgestomet and an estrogen in conjunction with a norgestomet implant in heifers and cows. Theriogenology, 10, 201-213.

PACE M. M., SULLIVAN J. J., 1980. Effect of synchro-mate-B (SMB) and calf separation on beef cattle estrus and pregnancy rates. J. anim. Sci., 51, suppl. 1, 312.

PETERS A. R., 1984. Reproductive activity of the cow in the post-partum period. I. Factors affecting the length of the post-partum acyclic period Br. vet. J., 140, 76-84.

PETERSON L. A., MARES S. F., HENDERSON E. A., DAVENPORT M. E., 1979. Effect of calf separation time on pregnancy rates of cows synchronized with synchro-mate-B (SMB). J. anim. Sci., 49, suppl. 1, 326-327. 
RANDEL R. D., 1981. Effect of once daily suckling on post-partum interval and cow calf performance of first-calf brahman $X$ hereford heifers. J. anim. Sci., 53, 755-757.

SMITH M. F., BURREL W. C., SHIPP L. D., SPROTT L. R., SONGSTER W. N., WILTBANK J. N., 1979. Hormone treatments and use of calf removal in post-partum beef cows. J. anim. Sci. 48, 1285-1294.

SMITH T. E., VINCENT C. K., 1972. Effects of early weaning and exogenous hormone treatment on bovine post-partum reproduction. J. anim. Sci., 35, 1228-1237.

TERVIT H. R., SMITH J. F., GOOLD P. G., JONES K. R., VANDIEN J. J. D., 1982. Reproductive performance of beef cows following temporary removal of calves. Proc. N.Z. Soc. anim. Prod., 42, 83-85.

THIMONIER J., 1978. L'activité ovarienne chez les bovins. Moyens d'étude et facteurs de variations. Ann. Méd. vét., 122, 81-92.

WALTERS D. L., BURREL W. C., WILTBANK J. N., 1984. Influence of exogenous steroids, nutrition and calf removal on reproductive performance of anoestrous beef cow. Theriogeno$\log y, 21,395-406$. 